\section{A new edition of a must have handbook for genetic counsellors \\ Practical genetic counselling $(2004)$}

Edited by Peter S Harper, Arnold, Hodder Headline Group, London, 6th Edition. ISBN $034081196 \mathrm{X}$.

...........

Hans-Christoph Duba

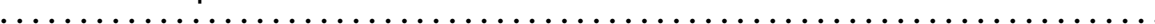

European Journal of Human Genetics (2005) 13, 1098.

doi:10.1038/sj.ejhg.5201461

T his recently published 6 th edition of Practical Genetic Counselling edited by PS Harper represents an updated guidance through the general principles and philosophy of genetic counselling. The content of the book is divided into three sections. In the first part, an introduction on genetic counselling is given. Following this introduction, there is detailed information on genetic counselling in Mendelian and common non-Mendelian disorders. There is a chapter describing the risks in specific groups of chromosomal disorders. The chapter on molecular genetics and genetic counselling covers the implications of the human genome project for clinical genetic practice and shows, by touching the more and more important aspects of molecular genetics, the inclusion of molecular techniques into the management of families with genetic disorders. The rapidly increasing knowledge of the human genome and its impact on the diagnosis of congenital malformations is shown in Chapter 6 - Dysmorphology and Genetic Syndromes. The statement that clinical geneticists have now a special advantage in the diagnosis of malformation syndromes by combining clinical genetic skills with the new molecular methods shows the farsightedness of the editor. The chapter on carrier testing and genetic prediction explores the range and limitations of tests of carrier detection and attempts to show how the information can be used in conjunction with other genetic and clinical data to make an accurate prediction of the risk to be a carrier. The chapter about prenatal diagnosis and related reproductive aspects discusses the influence of ultrasound and new laboratory methods on prenatal diagnosis and genetic counselling as well as the serious limitations of preimplantation genetic diagnosis. Chapter 9 deals with special issues in genetic counselling such as consanguinity, paternity and relationship testing, twins and legal problems in medical genetics. The chapter about the genetic counselling clinic should encourage clinicians to regard genetic counselling as a integral part of the management of patients and their families, and dispels the view that all genetic problems must be referred to a specialist clinic at all times - a point of view that varies in different European countries. The second part of the book gives the reader a precious tool as to how to manage genetic counselling regarding genetic diseases of special organ systems, especially neuromuscular diseases, central nervous system disorders, disorders of mental functions, disorders of bone and connective tissue, oral and craniofacial disorders, the skin, the eye, deafness, cardiovascular and respiratory disorders, the gastrointestinal tract, renal disease, endocrine and reproductive disorders, inborn errors of metabolism, disorders of blood and immune function, genetic risks in cancer and environmental hazards. Chapter 25 - genetic risks in cancer - deals with the increasing possibilities of recognising many of the high-risk cancer forms through identifying the genes involved. Part three includes the more general aspects of genetic counselling population aspects of genetic counselling, genetics and society. In the appendix, useful Internet addresses and a list with probably unfamiliar genetic terms are provided.

Designed as a compendium, the 6th edition is still small in size and of easy portability in spite of the increased coverage of the implications of the human genome project for clinical genetic practice, the expanding societal implications of human genetics, and the increased coverage of genetics and common diseases, including cancer genetics. The layout of the book has been improved and modernised. Only the index could be of more clearness by giving those page numbers in bold type, which primarily discuss the searched term.

In summary, the 6th edition of Practical Genetic Counselling presents as a musthave handbook for clinical medical geneticists, specialists who do genetic counselling and physicians who want to get a thorough insight into the complexity of genetic counselling

Hans-Christoph Duba is at the LandesFrauen-und Kinderklinik,

Humangenetische Untersuchungs-und Beratungsstelle, Lederergasse 47, Linz A-4020, Austria.

E-mail: hans-christoph.duba@gespag.at 